bruit at the apex, conducted to the left for a short distance: I'he pulmonary second sound rvas not notably accentuated there was no cyanosis. When the test was applied he had there was no cyanosis. When the test was applied he had
improved under rest and treatment. The apex beat was to $\frac{3}{4}$ in. within the nipple line. The heart sounds were closed, and other physical signs remained the same after as before exercise. There was a very decided nervous element in the case, and $I$ was inclined to regard the dilatation of the heart as caused by persistent irregular tachycardia. The reactions on both occasions would seem to fall into the ctass of "good" reactions; but one has to remember that the response would probably not have been good to more searching tests. In this case I did not feel justitied in applying more severe tests.

\section{Heart Strain.}

The Leipzig school-De la Camp, Krauss, Moritz, and their followers-maintain that it is quite impossible to push the healthy heart and great arteries beyond the limits of their reserve by any muscular effort. This may or may not be true of the healthy heart, but it would be absurd to contend that it applies to a heart of which the myocairdium has been previously damaged. Even Zuntz and Schumberg, of the German school, recognize "march dilatation of the right heart" in soldiers.

Apropos of dilatation of the right heart, one physical sign which I have found present in at least 40 or 50 per cent. of D.A.H. cases seems significant. 'The superficial cardiac dullness is extended upwards in the left-parasternal, often as far upwards as the upper end of the third left intercostal space. Since almost the whole of the anterior surface of the heart is formed by the right auricle and ventricle, and the right ventricle lies behind the third, fourth, and fifth left intercostal spaces, we can hardly resist the conclusion that this physical sign is produced by dilatation of the right ventricle. When we consider with it the fact that murmurs in D.A.H. cases are so frequently heard in the third, fourth, and fifth interspaces between the nipple line and the sternum, usually close to the sternum, we seem bound to give them careful consideration. It is unjustifiable to dismiss them lightly as "accidental" or "haemic" murnurs. "Dis. ordered action of the heart " is not necessarily a harmless transitory condition.

Captain MeCarthy, R.A.M.C., who studied the condition in a number of cases, found that the prognosis was not good.4 In the majority of cases the patients returned to hospital again and again, until they were invalided ont of the service. His impression, formed by following up the history of men invalided, was that many cases ended in valvular disease.

In dealing with D.A.H. cases our prime object is to form as shrewd an estimate as possible of the quality of the muscle fibre with which we are dealing in each individual case. The previous history, therefore, is of great importance, and the rôle played by past attacks of the infections febrile diseases in causing degeneration of the myocardium should not be forgotsen. ${ }^{5}$ It is well to bear in mind also Nieneyer's dictum that there are probably many forms of degeneration of the myocardium which cannot be detected by the microscope.

Even those with the greatest experience of "heart strain" have to content themselves with hypotheses as to its pathology. Sir Clifford Allbutt suggests that a molecular change, probably not to be detected by the microscope, may occur in the cardiac muscle tibre leading to diminished resilience.

\section{Size of Heart during Exercise.}

In support of the (rerman school's theory that the liealthy heart cannot be dilated by muscula: exercise, radiographer's now maintain that the heart does not increase in size duriug exercise; on the contrary, the right ventricle is not dilated, and the left ventricle actually diminishes in size.

After reading pages of controversy on the subject one feels that there is little, if any, ground for contention. Physiologists have never maintained that the hear't dilates and remains dilated throughout muscular exercise. They tell us that during the earliest stages, before velaxation of the arterioles occurs, the right heart takes the strain off the left ventricle by accommodating an increased output of venous blood from the muscles. But in a well-trained, "hardened" athlete or soldier this is only a passing phase. 'The increased volume of blood is dealt with by $(a)$ in. creased alveolar capacity of the lungs; $(b)$ development of the thoracic muscles; (c) by fall of peripheral resistance, which enables the blood to flow more easily through the left ventricle. Leonard Hill found that arterial pressures exceed ordinary levels for the first ten or fifteen minutes of exercise; then, as the periphery opened out, they fell.

In short, there is no need to insist on either great hypertrophy of the left ventricle or perilous dilatation of the right ventricle during exercise. Both speed and endurance would seem to depend not more on hypertrophy and increased capacity than on efficient correlation of the many mechanisms devised to assist the heart and to diminish its work. In other words, men do not run with their hearts alone, but with their hearts, lungs, thoracic muscles, and peripheral vessels, especially those of the skin.

\section{Evidence Against the Value of the Exercise Blood Pressure Test.}

Hirschfelder, after discussing the work of Graupner and Baur, advances the following objections to the test: ${ }^{6}$

1. That the blood pressure in trained athletes falls during mild exercise, exactly as it does in broken compensation; also that it falls when the "second wind" is acquired and while the person's functional power is increasing rather than decreasing. 2. The greatest rises of blood pressure occur in old and feeble
persons, whom the exercise brings near to the border line of cardiac overstrain.

3. In persons in whom the blood pressure falls as a result of the test exercise, the general symptoms, respiratory distress tachycardia arrhythmia, etc., are more than sufficient evidenee that the patient's strength has been overtaxed. These simple signs are more delicate indices and less ambiguous than the changes in blood pressure.

Following De la Camp, Schott, Moritz and others, he concludes :

The only true criterion of cardiac efflciency is whether a given strain causes it (the heart) to diminish in size (increase in tonicity = stimulation) or to dilate (decrease in tonicity = overstrain).

For my part I am already convinced that none of these objections should induce us to abandon the test, which seems distinctly valuable in two directions: (1) as confirmatory evidence of the results of ordinary physical examination in some cases; (2) as an additional test in other cases in which the diagnosis between functional disorder and organic disease of the myocardium is doubtful.

It is noteworthy that even. Hirschfelder, after all his destructive criticism, commits himself to the following statement:

This does not mean that the exercise tests are unimportant. On the contrary, they are of the greatest possible value; and no change in the patient's mode of life during convalescence or during after-life should be undertaken without them.

$$
\text { REFERENCES. }
$$

1 Strickland Goodall : Estimation of Myocardial Efficiency, Bartrom

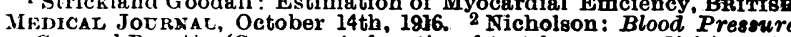
in General Practice (Graupner's functional test for myocarditis), pp. 69 , 70. 3 Allbutt and Rolleston's System of Medicine (1909), vol. vi: Over stress of the Heart, pp. 129-146. Allbutt and Rolleston (vide Reference 3 ). ${ }^{5} \mathrm{G}$. Lambert: Medical Chronicle, May, 1907, Certain Effects of Febrile Diseases upon the M
of Heart and Aorta, pp. 129-146.

\section{PULMONARY FAT EMBOLISM AND ITS RELA- TION TO TRAUMA'IIC SHOCK.}

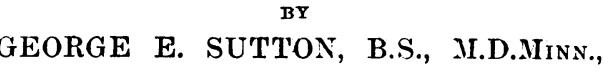 \\ Captatn M.R.C.
}

ON my arrival at a casualty clearing station in France last year I was greatly impressed by the type of cases of so-called shock, which closely resembled cases of pulmonary fat embolism I had seen with Bissell at the Mayc chinic. During the last ten months I have been able to investigate a considerable number of cases whose clinical condition was assimilated to "shock."

It is well known to those who have had experience in the resuscitation wards in forward areas that there is a type of wounded admitted characterized by the following features :

Cyanosis, moderate varying to deep.

Pulse small, easily compressible, and increased. in frequency.

Breathing sometimes laboured and increased in rate.
Extremities cold.

Deliriam of varying degree.

It will have been noticed also that there is no appre. ciable or sustained response to measures of resuscitation- 
warmth, elevation, rest, and intravenous or subcutaneous stimulation. Within a few hours the patient succumbs; the diagnosis " shock."

In these cases post-mortem examination invariably reveals pulmonary fat emboli which can be demonstrated grossly. If this fails, sections of lung tissue should be stained for fat. A typical and interesting case may be cited:

In the reception room at a casualty clearing station 1 saw a patient admitted with a gunshot compound fracture of the left femur and a gashing wound of the left abdominal wall. In addition to the symptoms resembling shock he was in a state of mild delirium which could not be accounted for by blood loss.

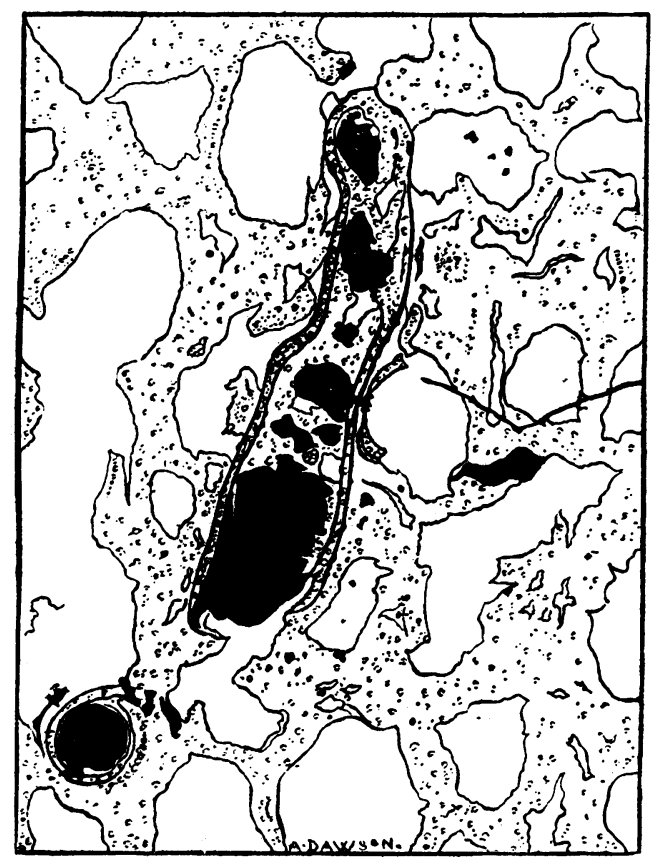

Typical fat embolism. Section of lung tissue $(\times 57)$ from case of compound fracture of femur.

This was four hours after injury. The patient was sent to the resuscitation ward, where he was treated by elevation of the loot of the bed, warmth, rest, and intravenous injection of sodium bicarbonate solution (2 per cent.). He died eight hours later.

Autopsy revealed no macroscopic fat, but on staining sections ol lung tissue the capillaries were found riddled with fat. I suspected this case of having a pulmonary fat embolus because of the otherwise unaccountable delirium.

Examination of a considerable number of battle casualties which have terminated fatally at a casualty clearing station has led me to the conclusion that 10 per cent. have pulmonary fat emboli demonstrable by gross methods. I am convinced that this percentage would be increased (to what extent it is impossible to say) if sections of lung tissue were stained for fat in all cases.

Cases in which Fat Emboli have been Found.

I. Fractures.

1. Compound fracture of long bones. (a) Single. (b) Multiple.

2. Fracture of skull with destruction of brain tissue.

3. Fracture of ribs.

II. Wounds involving fatty tissue.

1. Penetrating abdomen with maceration of omentum.

2. Large wounds of buttock and trunk.

III. Wounds penetrating the abdomen, with laceration of the liver.

In regard to fractures of long bones a good deal may be said. It has been particularly noticed that in compound fractures reaching a casualty clearing station with splints imperfectly applied, and where, consequently, immobility was not complete, symptoms resembling "shock" were invariably present.

I have seen pulmonary fat embolism more often following a compound fracture without comminution than with comminution.

During a recent crisis, when patients had to be evacuated to casualty clearing stations more hurriedly than in ordinary times a number of cases were received, many in a profound state of "shock." The blood loss in some was not great, as evidenced by the condition of their clothing and stretchers. Amongst these, few showed any response to resuscitation measures. In this series $F$ examined the lung tissue of eight cases, and found pulmonary fat embolism in six. Time would not permit investigation of all.

Relation of Pulmonary Fat Embolism to Shock.

Warthin has shown that repeated injections of olive oil into the right auricle of a dog causes a marked fall of carotid pressure and a marked rise in jugular pressure. Fischer has shown that when olive oil is injected into the vein of a rabbit more than 60 per cent. soon lodges in the lung. His explanation of this is based on the elasticity of pulmonary capillaries, decreased tissue pressure surrounding the pulmonary capillaries, and negative alveolar pressure.

Gauss demonstrated that, with constant factors, the addition of 10 per cent. olive oil increased the viscosity of blood 200 per cent.

Experiment by Gauss substantiated by Bissell.

Time required for $1 \mathrm{c.cm}$. of fluid to pass through the capillary tube under constant pressure of $70 \mathrm{~mm}$. of mercury, and constant temperature of $24.5^{\circ} \mathrm{C}$. :

\begin{tabular}{lll|ll|ll}
\hline & & \multicolumn{2}{|c|}{ Alone. } & Plus Olive Oil. \\
\hline Salt solution ... & $\ldots$ & $\ldots$ & 33 seconds & 100 seconds. \\
Ascitic fluid ... & $\ldots$ & $\ldots$ & 45 &. & 130 & \\
Human blood serum & $\ldots$ & 57 &. & 180 &. \\
Human blood citrated & $\ldots$ & 160 &. & $480 \quad$, \\
\hline
\end{tabular}

Bissell states: "It being known that pulmonary fat embolism, both in man and experimentally in animals, canses a decrease arterial pressure and increased venous pressure even to fatal termination, it is reasonable to presume that venous blood rich in fat would offer additional resistance to passase through capillaries due to its increased viscosity. Certainly, it cannot be denied that in the lung capillaries, where fat is accumulated

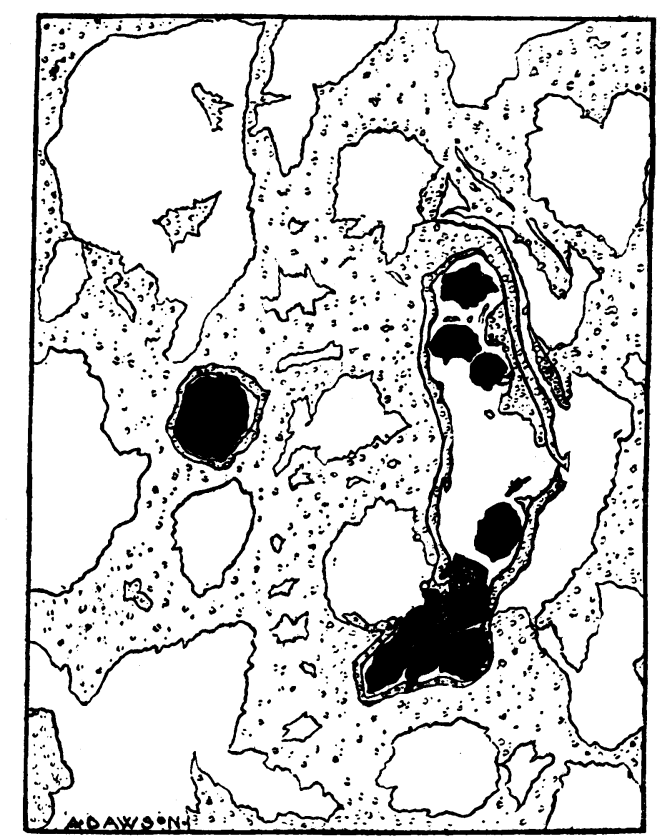

Typical fat embolism. Section of lung tissue $(\times 57)$ frow case of compound fracture of femur.

as by repeated injections, the viscosity of the blood must be greatly increased.

To quote again from Bissell: "The diagnosis of fat embolism, clinically, is based on observations of phenomena produced by distinct, unmistakable lesions grossly demonstrable at necropsy, while the clinical diagnosis of shock is based on observations of signs and symptoms exactly duplicated in pulmonary fat of signs and symptoms exactly duplicated in pulmonary fat plained in the examination of dead bodies unless haemorrhage plained in the examination of dea,

To carry the argument one step along, if our unsuccessful cases of slock are due to an acidosis-a prominent opinion to-day-liave we not all the factors in pulmonary fat embolism, in varying degree, to produce this acidosis- 
namely, a tremendous increase of blood viscosity, high venous pressure, slowing of blood flow, and deficient blood aëration?

By the above statement I do not mean to assert that all fatal cases of shock are due to pulmonary fat embolism, but that the physical and chemical properties of lipaemic blood are such that acidosis cannot be avoided, and it is still to be proven whether the terminal mechanism is not the same.

The occurrence of pulmonary fat embolism in the war area is much more frequent than has been suspected, owing, probably, to lack of thorough examination of lung tissue for evidence of fat.

Associated with pulmonary fat embolism disseminated fat is found in the other tissues of the body, included in the capillaries. This undoubtedly accounts for the delirium so frequently associated with this condition.

Examination of Lungs for Pulmonary Fat Embolism.

Use a glazed earthenware plate or platter washed thoroughly with soap and water and rinsed; the knife is cleaned in the same manner.

Wipe the surface of the lung clean with a dry towel. With one long sweep cut in to the lung, then, free from contamination with fatty tissue, express some of the blood from the cut surfaco. Allow surface. Allow this blood to flow slowly in a thin stream over the platter. If there is fat present it will appear as little globules on the surface of the expressed blood. Experience on the detection of small amounts of fat an easy matter.

\section{Treatment.}

1. Prophylactic treatment consists in fixation of fractures with extension where possible; early and efficient surgical interference for fractures; at operation on wounds in volving fatty tissue it is imperative to secure careful and complete haemostasis, particularly the ligation of the nonbleeding proximal ends of veins as well as the bleeding distal ends.

2. Active treatment is only symptomatic. Small amounts of sodium bicarbonate (2 per cent.) are helpful. The administration should be regulated by the condition of the right heart. Transfusion of blood in small amounts may be useful.

\section{Conclusions.}

Pulmonary fat embolism is much more frequent in the war zone than was formerly thought.

A reasonably accurate diagnosis of pulmonary fat embolism can be made.

Cases of pulmonary fat embolism do not respond readily to measures of resuscitation.

The ultimate cause of death in pulmonary fat embolism and in shock may be the same-namely, acidosis.

\section{ReFERENCes.} Fischer, B.: Verhandl. d. deutsch, path. Gesellsch., 1914, xviii.

\section{THE SURGICAL CURE OF UTERINE PROLAPSE. BX}

\section{HUGH P. COSTOBADIE, F.R.C.S.EDIN.}

THe surgery of the present war is occupying the minds of all, and so rapid has been its progress in many respects that our whole attention is required to keep in touch with its various developments. As yet, however, but little of the new work has found an application to general surgery, and it is doubtful whether, excluding orthopaedics, accidental injuries, and possibly the surgery of the chest, any other branch is likely to benefit at all. On the other hand, there are a few surgical conditions, apart from wounds, which have become somewhat more common during this period, and therefore more experience has been gained in them. Of these, hernia, both in the male and female, and prolapse of the uterus are being more frequently encountered. Alternative operations for the former condition are being published and discussed, and the results are being more readily and carefully followed, so that some improvement is likely to accrue in the surgical treatment of this condition. Unfortunately uterine prolapse has not received so much attention.

The following paper has been written to describe what are probably the best operations for the cure of prolapse practised to-day. Though very much pre-war operations, they are not as well known as they deserve to be, nor is their reputation likely to spread at a time when pressure of work tends to make men adhere to their own methods, unless their attention is especially called to other measures.

That prolapse should increase can be readily understood, and it may chiefly be accounted for by the following factors:

1. Great increase of women workers.

2. Heavier kinds of employment.

3. Less food, especially fats.

4. Increase of wage, which keeps the woman at work even when she is ill and brings her back to work before she is recovered, especially from her confinements.

In other words, it more frequently happens to-day that increased intra-abdominal pressure through heavy work is enabled to take such effect upon generally weakened uterine supports in a woman whose occupation keeps her standing and whose pelvic floor has either been recently injured and has not had time to recover, or has at some previous date become impaired, that prolapse results.

-To treat this, rubber rings can scarcely ever be recom mended, and can only occasionally be used, either as a temporary measure, or for a patient upon whom operation is contraindicated. In the woman worker they very soon become irritating because they lack the necessary atten tion she is unable to give. Operation should therefore be advised in all these cases, whether they are within the child-bearing age or no.

Fixation and suspension of the uterus for prolapse is still practised by many surgeons, but the results with patients on whom I performed this operation some time ago have not been altogether satisfactory, nor have the results been much better in those fow cases I have seen where suspension was performed for the same purpose by other surgeons. In cornbination with some form of colporrhaphy the results are better. Hysterectomy need not be considered, though even recently I heard of hysterectomy alone being performed for prolapse, with, of course, a subsequent complete eversion of the vaginal canal.

Of the vaginal operations still performed for the definite cure of prolapse, the transposition operation largely used in America, and extensive anterior and posterior colporrhaphies, as practised in Manchester and elsewhere, are the only methods by which a cure can be almost definitely promised. The American "transposition" operation con sists of freeing the uterus and bladder, and using the former and the cervix, or a necessary portion of them, after the remainders have been excised; to give a firm sup port to the bladder. A posterior colporrhaphy or perineor rhaphy is then performed. The published results of this operation are good, but it has several obvious drawbacks which I think are not possessed by the method of anterior and posterior colporrhaphy which $I$ am about to describe. I first saw these colporrhaphies performed by Professor Donald and Dr. Fothergill of Manchester, and have since carried them out according to their technique with uniformly good results. The operation in the case of com. plete prolapse, with the almost always present elongation of the cervix, consists of amputation of the cervix by a circular method, or by means of anterior and posterior flaps, followed by a large anterior and posterior colporrhaphy. Dr. Fothergill carries out the amputation and anterior colporrhaphy by means of one incision. Dr. Donald amputates the cervix by means of anterior and posterior flaps, and then follows the anterior colporrhaphy, a separate step entirely. Either operation may be per. formed according to the requirements of each individual case. Usually I prefer to amputate by means of flaps when the patient is of child-bearing age, but after that time I use the circular method, where, should stenosis of the canal follow, it would not be of so much importance. I perform this operation as follows, which is, I believe, as nearly as possible the method employed by the Manchester surgeons.

The patient having been prepared, is placed in the lithotomy position; the vagina is freely flushed out with a solution of fysol, followed by perchloride. It is then carefully dried out with swabs and washed with spirit and the surrounding surface painted with iodine. The anterior lip of the cervix is grasped with vulsellum foreeps and $a$ sound is passed to give an accurate measure of the amount of cervical elongation. The cervix is then dilated sufficiently to admit the flushing. curette, and the interior of the nterus is carefully curetted. The posterior lip is now grasped with second pair of vilsella and posterior lip as far as possible, overting the vaginal eanal aimost completely. 